\title{
THE GROWTH OF LINEAR PERTURBATIONS OF ADIABATIC SHOCK WAVES
}

\author{
DONGSU RYu AND ETHAN T. VISHNIAC ${ }^{1}$ \\ Department of Astronomy, University of Texas \\ Received 1986 March 21; accepted 1986 August 8
}

\begin{abstract}
We consider the linear theory of the dynamic instability of strong plane-parallel or spherical adiabatic blast shock waves in a medium with initially uniform density. We obtain the principal frequency eigenvalues as a function of transverse wavelength for several values of the adiabatic index. We find that the plane-parallel and spherical shocks are overstable for the adiabatic index $\gamma \lesssim 1.2$. We discuss the astrophysical implications of these results. These detailed eigenvalue and eigenmode results provide an opportunity to test the accuracy of three-dimensional numerical models of shock wave evolution.
\end{abstract}

Subject headings: hydrodynamics - instabilities - shock waves

\section{INTRODUCTION}

For several decades, the expansion of a strong shock following the instantaneous release of large amounts of energy has been studied extensively. It is generally understood that three different stages in the expansion may be distinguished (Spitzer 1978). In the first stage, the outward moving shock sweeps out an amount of ambient gas roughly equal in mass to the ejecta from the initial explosion. In the second stage, the temperature of the shell behind the shock front becomes so high that radiative loss of energy can be neglected at a first approximation, and the shock evolves into an adiabatic shock with conserved total energy. In the third stage, radiative cooling becomes important, the temperature falls rapidly, and the shock becomes effectively an isothermal shell.

The evolution of the various shocks in the interstellar medium or in the intergalactic medium was discussed by several authors in order to explain star formation (Elmegreen and Lada 1977; Elmegreen and Elmegreen 1978; Welter and Schmid-Burgk 1981; Welter 1982) or galaxy formation (Ostriker and Cowie 1981; Ikeuchi 1981; Ikeuchi, Tomisaka, and Ostriker 1983; Bertschinger 1983). An important consideration in these discussions is the stability of the shell behind the shock front in various stages of its evolution.

Adiabatic blast shocks can be used as approximations in dealing with shocks produced by large explosions, because they yield a known analytic solution described by a similarity flow. Also, several important properties of their solution (such as age and initial energy release) can be directly inferred from observable quantities. The similarity formalism demands that flow quantities depend only on a single dimensionless parameter involving length and time scales. In the case of the adiabatic blast shock, this requires that it have central symmetry, that the density of the ambient gas be describable by a power law in radius, and that the external pressure be negligible with respect to the internal pressure behind the shock. The similarity formalism can be expected to be an excellent approximation, provided that it is applied only in the region far from the spatial and temporal boundaries of the problem and that it contains an adequate description of the physics. For the case of the adiabatic blast shock, the first condition implies that it can be applied only at sufficiently late times when the explosion loses the details of initial conditions. The second condition implies that energy loss by radiation must be unimportant.

The stability of blast shocks has been studied widely, but a complete theoretical analysis has not yet been done. Erpenbeck (1962) studied the stability of a steady plane-parallel shock dividing the space into two homogeneous regions and proved it to be at least exponentially stable against a rippling of the shock front. Isenberg (1977), Cheng (1979), and Newman (1980) discussed the stability of a spherical adiabatic blast shock in a medium with a density distribution of a power-law form and showed that it is not unstable against purely radial perturbations. However, no analytic demonstration of the stability exists. Bernstein and Book (1980) and Gaffet $(1984 a, b)$ considered the stability of a Primakoff blast shock in which the velocity of similarity flow behind the shock is proportional to a dimensionless similarity parameter. Bernstein and Book showed that it is stable against perturbations with any wavelength, but Gaffet claimed that it can be proved only to be radially stable. However, it is generally believed, on the basis of the cumulative results of gasdynamic experiments and numerical simulations, that the adiabatic blast shock is stable for the usual values of the adiabatic index (Newman 1980).

Vishniac (1983) examined the linear theory of the stability of a spherical blast shock in a medium with uniform density against dynamic and gravitational perturbations when the thickness of the dense shell behind the shock is small compared to the wavelength of the perturbation and the radius of the shock front. For dynamic perturbations, he proved the overstability of perturbations of the thin shell, but he left unsolved the exact effect of the finite thickness of any realistic shell. On physical grounds, following the results of Erpenbeck (1962), he suggested that the overstability is stabilized for the wavelengths corresponding to $k h>1$, where $k$ is wavenumber and $h$ is the thickness of the shell. At slightly larger wavelengths, the fragmentation is expected to be maximally efficient. Vishniac showed that an isothermal shock is overstable for a range of wavelengths given by

$$
6.4<k R<3 M^{2} \text {, }
$$

\footnotetext{
${ }^{1}$ Presidential Young Investigator and Alfred P. Sloan Research Fellow.
} 
where $R$ is the radius of the shock front and $M$ is the Mach number of the shock front relative to the internal sound speed of the shell. A similer analysis, including cooling instabilities, can be found in Bertschinger, (1986). Vishniac also showed that an adiabatic shock is overstable for the value of the wavenumber in the range of

$$
20.4<k R<3 \frac{(\gamma+1)}{(\gamma-1)},
$$

where $\gamma$ is the adiabatic index. The adiabatic shock is overstable only if $\gamma \lesssim 1.3$. For gravitational perturbations, he showed that on cosmological time scales all such shocks are unstable, with density perturbations growing as power laws in time; but whenever dynamic instability is at work, gravitational instability can be neglected in the fragmentation of the shock.

It is of considerable physical interest to calculate the wavelength of maximum overstability and the minimum wavelength of overstability for a realistic shell with a finite thickness. In the real world, the shell should be produced by a radiative shock. But such a radiative shock cannot be represented by a similarity formalism, and we, therefore, approximate the radiative shell as the adiabatic shell with $\gamma$ approaching 1 . We consider a detailed analysis of the linear theory of the dynamic instability of plane-parallel and spherical, adiabatic, blast shocks in a medium with uniform density. Gravitational instability is not considered, since it can be always neglected compared to dynamic instability. The viscosity and the magnetic field of the ambient gas are also ignored.

In $\S$ II we present the hydrodynamic equations which govern the gas flow motion of the adiabatic shocks and the similarity solutions of the unperturbed, strong, adiabatic shocks following Landau and Lifshitz (1959) for several values of $\gamma$. In $\S$ III we recover the frequency spectra of Vishniac (1983) for the shock with an infinitely thin shell which corresponds to $\gamma=1$, in order to compare them with those for the shock with finite thickness. In $\S$ IV the perturbation equations for $\gamma \neq 1$ are derived. In $\S \mathrm{V}$ the boundary conditions at the shock front and the origin are derived by methods similar to those used by Welter (1982). In $\S$ VI we discuss the numerical scheme used to solve the perturbation equations and give the frequency spectra for several values of $\gamma$. Finally, in $\S$ VII we discuss the implications of this work.

\section{UNPERTURBED SHOCK}

Let us consider the propagation of a strong adiabatic shock through an ideal gas. The equations which govern the similarity flow of the adiabatic shock are

i) the continuity equation:

$$
\frac{\partial \rho}{\partial t}+\nabla \cdot(\rho v)=0
$$

ii) the momentum equation:

$$
\rho \frac{\partial v}{\partial t}+\rho(v \cdot \nabla) v+\nabla p=0
$$

iii) the energy equation:

$$
\frac{\partial p}{\partial t}+\boldsymbol{v} \cdot \nabla p+\gamma p \nabla \cdot v=0 .
$$

Instead of the energy equation, we can use the entropy equation

$$
\left(\frac{\partial}{\partial t}+v \cdot \nabla\right) \log \frac{p}{\rho^{\gamma}}=0
$$

for the region behind the shock front.

If the shock is very strong, then, from the boundary conditions for the unperturbed shock at the shock front, the pressure $p_{2}$, the density $\rho_{2}$, and the gas velocity $v_{2}$ relative to the ambient gas just behind the shock front can be expressed in terms of the density $\rho_{1}$ of the ambient gas and the velocity of the shock front $u_{1}$ relative to the ambient gas as

$$
\begin{aligned}
& v_{2}=\frac{2 u_{1}}{\gamma+1}, \\
& \rho_{2}=\rho_{1} \frac{\gamma+1}{\gamma-1}, \\
& p_{2}=\frac{2}{\gamma+1} \rho_{1} u_{1}^{2} .
\end{aligned}
$$

\section{a) Plane-Parallel Shock}

Since the conserved quantity in a plane-parallel shock is the energy of the shock per unit area $\epsilon$, the shock is entirely determined by two quantities, $\rho_{1}$ and $\epsilon$. From these quantities and the two independent variables (the time $t$ and the rectangular coordinate $x$ ), we can form the dimensionless parameter $\xi$ of similarity flow

$$
\xi=x\left(\frac{\rho_{1}}{\epsilon t^{2}}\right)^{1 / 3} .
$$

\section{(C) American Astronomical Society - Provided by the NASA Astrophysics Data System}


Let us introduce the normalized quantities $\tilde{v}, \tilde{\rho}$, and $\tilde{p}$ and the normalized similarity parameter $\tilde{x}$ for the gas flow throughout the region behind the shock front which is defined by

$$
\begin{aligned}
& \tilde{v} \equiv \frac{v}{v_{2}} \\
& \tilde{\rho} \equiv \frac{\rho}{\rho_{2}}, \\
& \tilde{p} \equiv \frac{p}{p_{2}}, \\
& \tilde{x} \equiv \frac{\xi}{\xi_{2}} \equiv \frac{x}{x_{2}},
\end{aligned}
$$

where $\xi_{2}$ is the value of the similarity parameter at the shock front and $x_{2}$ is the distance of the shock front from the origin.

By integrating the equations of motion, we obtain the similarity solutions of the unperturbed plane-parallel shock

$$
\begin{aligned}
& \tilde{v}=\tilde{x} v^{\prime} \\
& \tilde{\rho}=\tilde{x}^{-3 v_{3} / v_{2}} v^{\prime-2 v_{3} / v_{2}}\left(3-2 v^{\prime}\right)^{-v_{1} v_{3} / v_{2}+v_{4}}\left(\frac{\gamma+1-2 v^{\prime}}{\gamma-1}\right)^{v_{5}}, \\
& \tilde{p}=v^{-2 v_{3} / v_{2}+2 / v_{2}+2}\left(3-2 v^{\prime}\right)^{-v_{1} v_{3} / v_{2}+v_{4}+v_{1} / v_{2}}\left(\frac{\gamma+1-2 v^{\prime}}{\gamma-1}\right)^{v_{5}+1}
\end{aligned}
$$

where

$$
\begin{aligned}
& v^{\prime 2}\left(3-2 v^{\prime}\right)^{v_{1}}\left(\frac{2 \gamma v^{\prime}-\gamma-1}{\gamma-1}\right)^{v_{2}}=\tilde{x}^{-3}, \\
& v_{1}=\frac{5 \gamma-4}{2 \gamma-1}, \\
& v_{2}=-3 \frac{\gamma-1}{2 \gamma-1} \text {, } \\
& v_{3}=\frac{1}{2 \gamma-1} \text {, } \\
& v_{4}=\frac{5 \gamma-4}{(2-\gamma)(2 \gamma-1)} \text {, } \\
& v_{5}=-\frac{2}{2-\gamma} .
\end{aligned}
$$

Figure 1 shows the normalized shock quantities as functions of $\tilde{x}$ for several values of $\gamma$.

\section{b) Spherical Shock}

In a spherical shock, the conserved quantity is the total energy of the shock $E$, and the dimensionless parameter $\xi$ of similarity flow becomes

$$
\xi=r\left(\frac{\rho_{1}}{E t^{2}}\right)^{1 / 5},
$$

where $r$ is the radial coordinate. The normalized similarity parameter is defined by

$$
\tilde{r} \equiv \frac{\xi}{\xi_{2}} \equiv \frac{r}{r_{2}},
$$

where $r_{2}$ is the radius of the shock front. An integration of equations of motion gives

$$
\begin{aligned}
& \tilde{v}=\tilde{r} v^{\prime}, \\
& \tilde{\rho}=\tilde{r}-5 v_{3} / v_{2} v^{\prime-2 v_{3} / v_{2}}\left[\frac{5(\gamma+1)-2(3 \gamma-1) v^{\prime}}{7-\gamma}\right]^{-v_{1} v_{3} / v_{2}+v_{4}}\left(\frac{\gamma+1-2 v^{\prime}}{\gamma-1}\right)^{v_{5}}, \\
& \tilde{p}=v^{\prime-2 v_{3} / v_{2}+2 / v_{2}+2}\left[\frac{5(\gamma+1)-2(3 \gamma-1) v^{\prime}}{7-\gamma}\right]^{-v_{1} v_{3} / v_{2}+v_{4}+v_{1} / v_{2}}\left(\frac{\gamma+1-2 v^{\prime}}{\gamma-1}\right)^{v_{5}+1},
\end{aligned}
$$



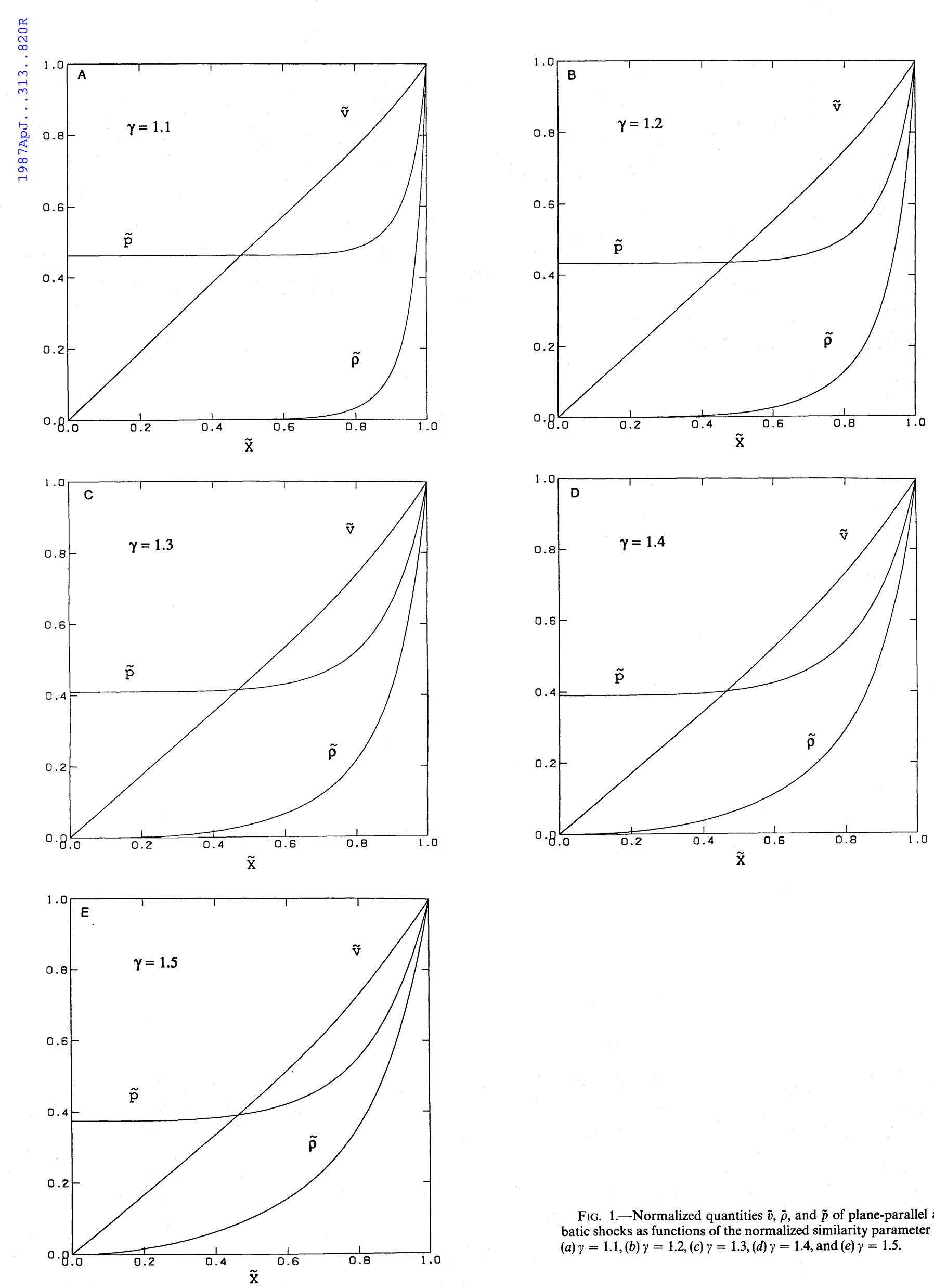

FIG. 1--Normalized quantities $\tilde{v}, \tilde{\rho}$, and $\tilde{p}$ of plane-parallel adiabatic shocks as functions of the normalized similarity parameter $\tilde{x}$ for (a) $\gamma=1.1,(b) \gamma=1.2,(c) \gamma=1.3,(d) \gamma=1.4$, and $(e) \gamma=1.5$. 

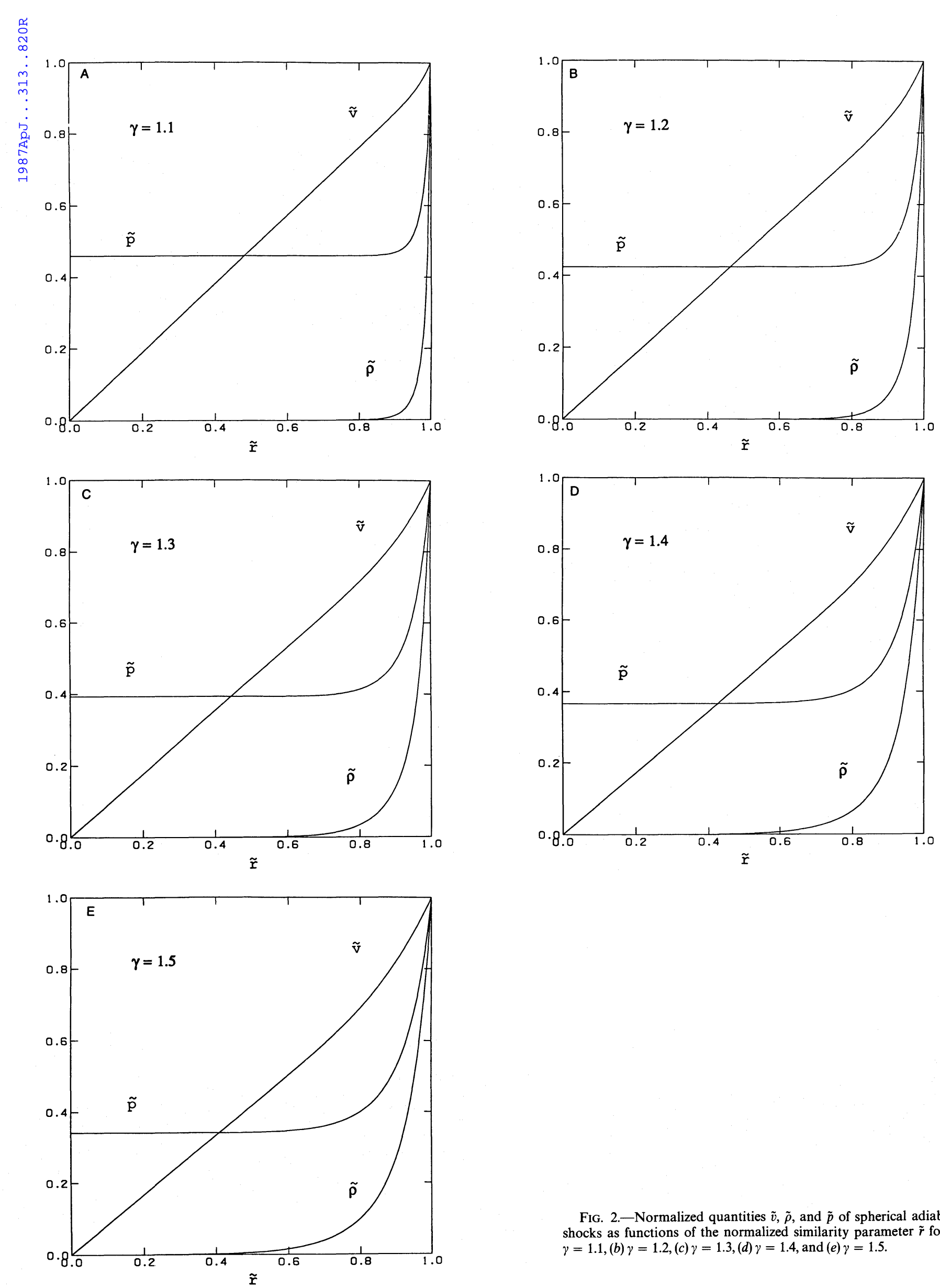

FIG. 2.-Normalized quantities $\tilde{v}, \tilde{\rho}$, and $\tilde{p}$ of spherical adiabatic shocks as functions of the normalized similarity parameter $\tilde{r}$ for $(a)$ $\gamma=1.1,(b) \gamma=1.2,(c) \gamma=1.3,(d) \gamma=1.4$, and $(e) \gamma=1.5$. 
where

$$
\begin{aligned}
v^{\prime 2}\left[\frac{5(\gamma+1)-2(3 \gamma-1) v^{\prime}}{7-\gamma}\right]^{v_{1}}\left(\frac{2 \gamma v^{\prime}-\gamma-1}{\gamma-1}\right)^{v_{2}} & =\tilde{r}^{-5}, \\
v_{1} & =\frac{13 \gamma^{2}-7 \gamma+12}{(3 \gamma-1)(2 \gamma+1)}, \\
v_{2} & =-5 \frac{\gamma-1}{2 \gamma+1}, \\
v_{3} & =\frac{3}{2 \gamma+1}, \\
v_{4} & =\frac{13 \gamma^{2}-7 \gamma+12}{(2-\gamma)(3 \gamma-1)(2 \gamma+1)}, \\
v_{5} & =-\frac{2}{2-\gamma} .
\end{aligned}
$$

Figure 2 shows the normalized shock quantities as functions of $\tilde{r}$ for several values of $\gamma$. A very rapid decrease of the density toward the origin is noticeable, and almost all the gas is in a relatively thin layer behind the shock front.

\section{STABILITY OF AN INFINITELY THIN SHOCK}

In equations (2.6) and (2.10), when $\gamma$ approaches 1 , the normalized shock quantities through the region behind the shock front will have the values

$$
\begin{aligned}
& \tilde{v} \approx \tilde{x}, \\
& \tilde{\rho} \approx 0, \\
& \tilde{p} \approx \frac{1}{2} .
\end{aligned}
$$

Hence the shock will become a sheet with infinite density. Inside this, the density is zero and the pressure is finite.

\section{a) Plane-Parallel Shock}

Using equations (2.1a), and (2.1b), let us consider three variables; the surface density

$$
\sigma \equiv \int_{x=0}^{x=x_{s}} \rho d x
$$

where $x_{s}$ is the distance of the sheet from the origin, the $x$-directional velocity of the gas $v_{x}$, and the tangential (say, the $y$-directional) velocity of the gas $v_{y}$ at the sheet. If we define the perturbation variables by

$$
\begin{aligned}
\delta(y, t) & \equiv \frac{\sigma-\sigma_{0}}{\sigma_{0}}, \\
\Delta x(y, t) & \equiv x_{s}-x_{2},
\end{aligned}
$$

where $\sigma_{0}$ is the average surface density and $x_{2}$ is the average distance of the sheet, we obtain the linearized perturbation equations

$$
\begin{aligned}
\frac{\partial \delta}{\partial t} & =\frac{1}{x_{2}} \frac{\partial \Delta x}{\partial t}-v_{0} \rho_{1} \frac{\delta}{\sigma_{0}}-\frac{\partial v_{y}}{\partial y}, \\
\frac{\partial^{2} \Delta x}{\partial t^{2}} & =-\delta \frac{\partial v_{0}}{\partial t}-\frac{2 \rho_{1} v_{0}}{\sigma_{0}} \frac{\partial \Delta x}{\partial t}, \\
\frac{\partial v_{y}}{\partial t} & =-\frac{\rho_{1} v_{0} v_{y}}{\sigma_{0}}-\frac{\rho_{1} v_{0}^{2}}{2 \sigma_{0}} \frac{\partial \Delta x}{\partial y},
\end{aligned}
$$

where $v_{0}$ is the unperturbed $x$-directional velocity of the sheet. Here we assume that there is no perturbation of quantities in the ambient gas. In equation (3.4c), the term which contains the pressure at the sheet is neglected, since the shock is infinitely thin. But for a shock with finite thickness $(\gamma \neq 1)$, the term containing the pressure cannot be neglected, and it must be considered through the third equation of motion, the energy equation, or the entropy equation. 
If we expand the spatial dependence of the perturbation variables in Fourier series, equations (3.4a) $-(3.4 c)$ may have solutions that are power-law in time. By assuming

$$
\begin{array}{r}
\delta \propto e^{i k y} t^{s}, \\
\frac{\Delta x}{x_{2}} \propto e^{i k y} t^{s}, \\
\frac{v_{y}}{v_{0}} \propto e^{i k y} t^{s}
\end{array}
$$

the secular equation of equations $(3.4 a)-(3.4 c)$ gives

$$
\left(s+\frac{4}{3}\right)\left(s+\frac{2}{3}\right)\left(s+\frac{1}{3}\right)^{2}+\left(\frac{2}{9} k x\right)^{2}=0,
$$

where the relations

$$
x_{2} \propto t^{2 / 3}
$$

and

$$
v_{0}=\frac{2 x_{2}}{3 t} \propto t^{-1 / 3}
$$

from equation (2.4) are used. Here we have one subtle difficulty in the plane-parallel shock case. In the Fourier expansion, we considered $k$ as a constant in equation (3.5). But in order that the solutions of the perturbation equation (3.4) exist, we have to consider $k x$ as a constant. Here $x=x_{2}$ is a function of time. This difficulty can be justified in the sense that if we are concerned with the short-term evolution of the perturbation with large $s$, we can recover the correct physical results. But as $s$ goes to a smaller value, the results are no longer physical. We will encounter the same difficulty in the plane-parallel shock with finite thickness. Of course, this difficulty does not appear in the spherical shock case. Figures $3 a$ and $3 b$ show the real and imaginary parts of the increasing modes of $s$ as functions of $k x$, and Figures $3 c$ and $3 d$ show those of the decaying modes of $s$. For a given value of $k x$, the shock is overstable if the real part of $s$ is positive. We can see from Figure $3 a$ that for $k x>15 \sqrt{3} / 8$ the shock is overstable with perturbations which grow in an oscillatory manner, and for smaller wavenumbers it is stable.

\section{b) Spherical Shock}

Following Vishniac (1983), let us define the perturbation variables by

$$
\begin{gathered}
\delta(\theta, \phi, t) \equiv \frac{\sigma-\sigma_{0}}{\sigma_{0}}, \\
\Delta r(\theta, \phi, t) \equiv r_{s}-r_{2},
\end{gathered}
$$

where

$$
\sigma \equiv \frac{1}{r_{s}^{2}} \int_{t=0}^{r=r_{s}} \rho r^{2} d x
$$

is the surface density and $r_{s}$ is the radius of the sheet. The third variable is $v_{T}$, the tangential velocity of the gas at the sheet. If we assume the perturbation variables have the spatial dependence in spherical harmonics and the time dependence in power law

$$
\begin{aligned}
\delta & \propto Y_{l m}(\theta, \phi) t^{s}, \\
\frac{\Delta r}{r_{2}} & \propto Y_{l m}(\theta, \phi) t^{s}, \\
\frac{v_{T}}{v_{0}} & \propto Y_{l m}(\theta, \phi) t^{s},
\end{aligned}
$$

the equations (2.1a) and (2.1b) give

$$
(s+1)\left(s+\frac{6}{5}\right)\left(s^{2}+\frac{11}{5} s+\frac{12}{25}\right)+\frac{36}{625} l(l+1)=0 .
$$

Here the relations

$$
r_{2} \propto t^{2 / 5}
$$

and

$$
v_{0}=\frac{2 r_{2}}{5 t} \propto t^{-3 / 5}
$$



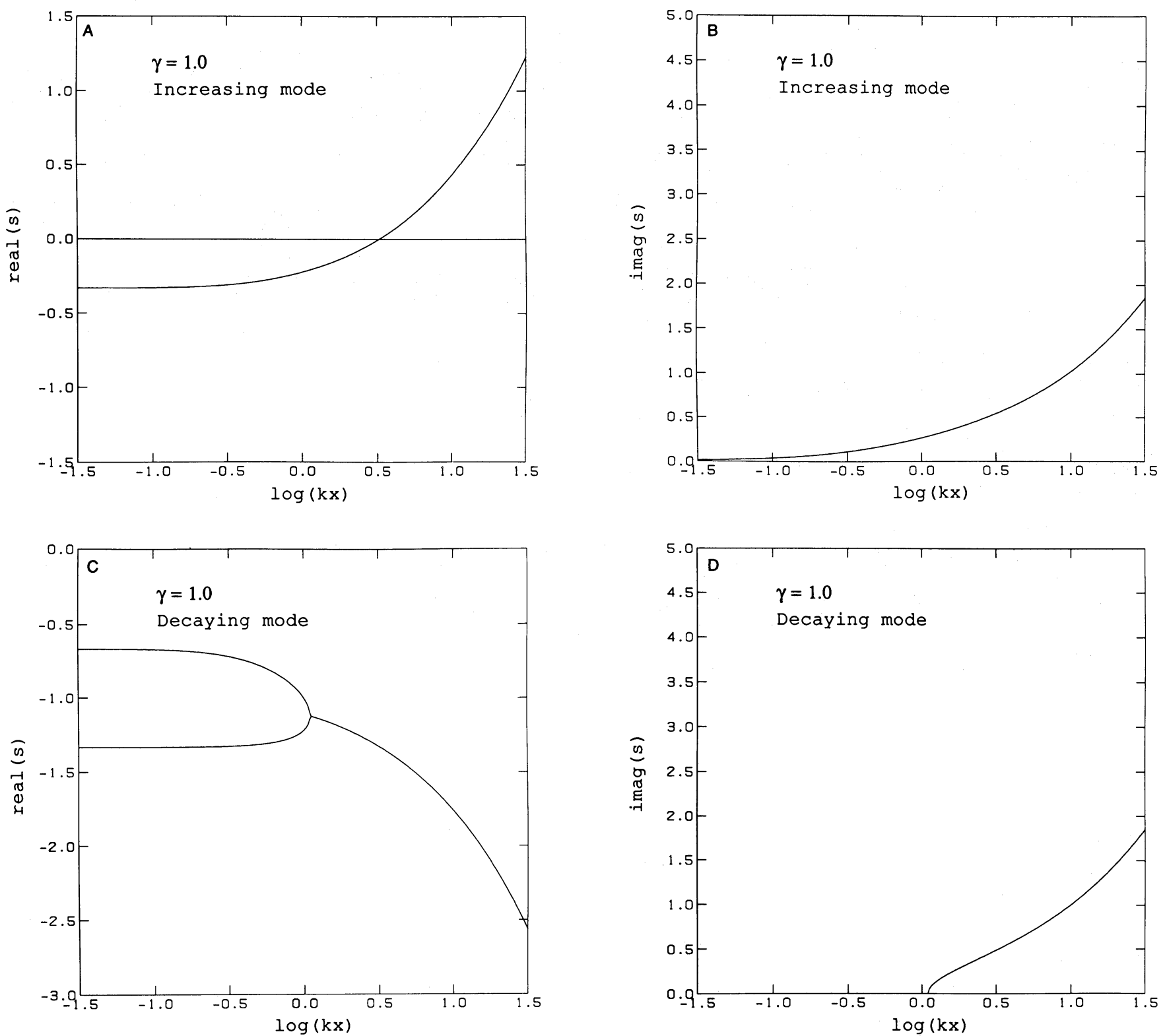

Fig. 3.-Real and imaginary parts of the dimensionless perturbation growth rate $s$ for a plane-parallel adiabatic shock with infinitely thin shell $(\gamma=1.1)$; $(a)$ and $(b)$ show the increasing mode, and $(c)$ and $(d)$ show the decaying mode.

from equation (2.8) are used. Figure 4 shows the real and imaginary parts of the increasing and the decaying modes of $s$ as functions of the discrete variable $l$. The infinitely thin shock is stable for $0 \leq l \leq 8$, because the matter accretion onto the shell make it stable against the perturbations with long wavelengths in one dynamical time (say, in the age of the shock).

\section{PERTURBATION EQUATIONS FOR A SHOCK WITH FINITE THICKNESS}

\section{a) Plane-Parallel Shock}

With the hydrodynamic equations of motion for an adiabatic shock (2.1a), (2.1b), and (2.2), let us consider the perturbation variables $\delta \boldsymbol{v}, \delta \rho$, and $\delta p$, which are defined by

$$
\begin{aligned}
& \delta v(x, y, t) \equiv v(x, y, t)-v_{0}(x, t) \hat{x}, \\
& \delta \rho(x, y, t) \equiv \rho(x, y, t)-\rho_{0}(x, t), \\
& \delta p(x, y, t) \equiv p(x, y, t)-p_{0}(x, t)
\end{aligned}
$$



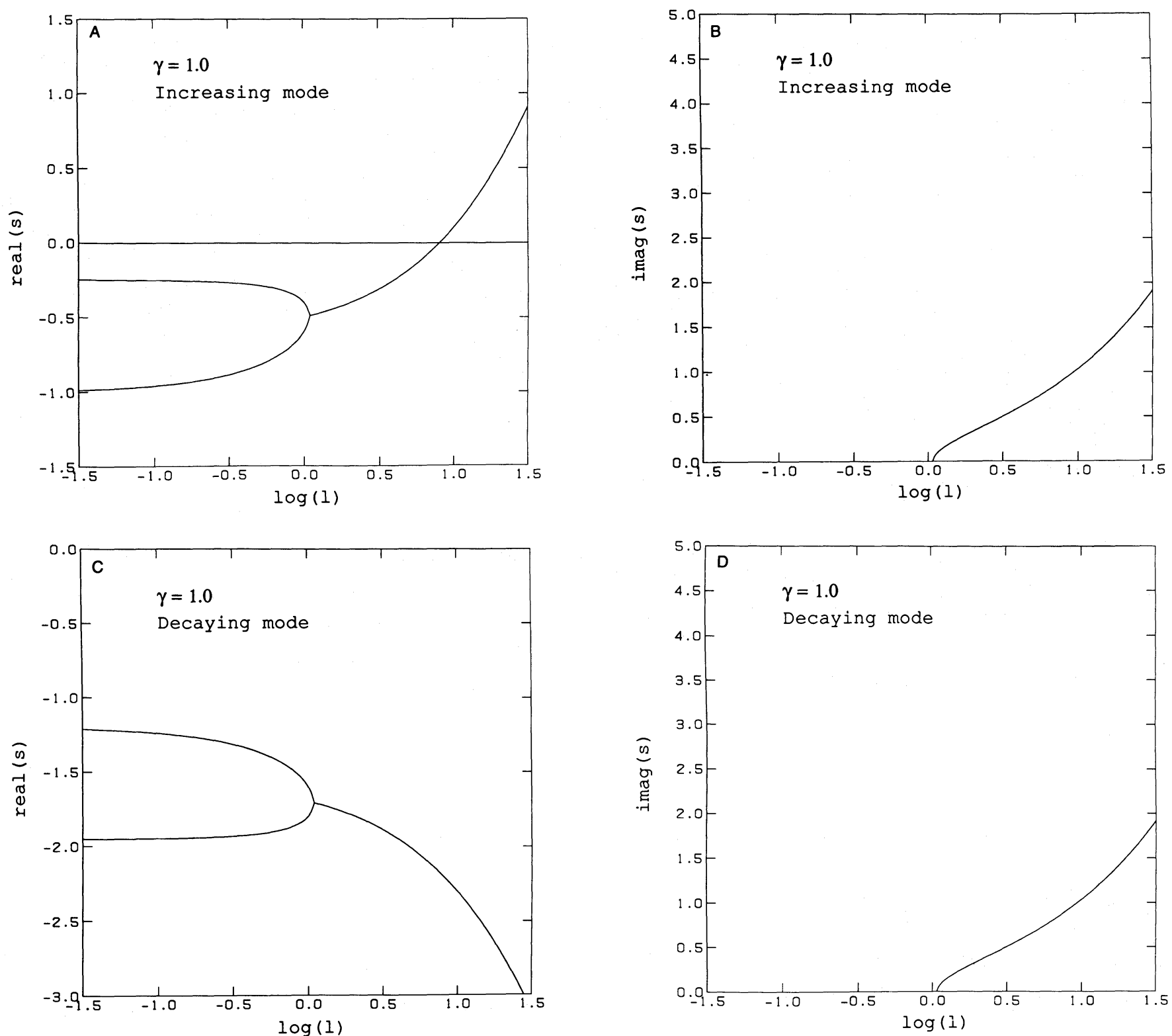

FIG. 4.-Real and imaginary parts of the dimensionless perturbation growth rate $s$ for a spherical adiabatic shock with infinitely thin shell $(\gamma=1.1) ;(a)$ and $(b)$ show the increasing mode, and $(c)$ and $(d)$ show the decaying mode.

Here $v_{0}, \rho_{0}$, and $p_{0}$ are the unperturbed quantities which are considered in $\S$ II. By linearizing the hydrodynamic equations about the perturbation variables, we obtain the perturbation equations

$$
\begin{aligned}
\frac{\partial \delta \rho}{\partial t}+\frac{\partial\left(v_{0} \delta \rho\right)}{\partial x}+\nabla \cdot\left(\rho_{0} \delta v\right) & =0 \\
\frac{\partial \delta \boldsymbol{v}}{\partial t}+v_{0} \frac{\partial \delta \boldsymbol{v}}{\partial x}+\frac{\partial v_{0}}{\partial x} \delta v_{x} \hat{\boldsymbol{x}} & =-\frac{1}{\rho_{0}} \nabla \delta p+\frac{\delta \rho}{\rho_{0}^{2}} \frac{\partial p_{0}}{\partial x} \hat{\boldsymbol{x}} \\
\left(\frac{\partial}{\partial t}+v_{0} \frac{\partial}{\partial x}\right)\left(\frac{\delta p}{p_{0}}-\gamma \frac{\delta \rho}{\rho_{0}}\right)+\delta v_{x} \frac{\partial}{\partial x} \log \left(\frac{p_{0}}{\rho_{0}^{\gamma}}\right) & =0 .
\end{aligned}
$$

If we define the normalized perturbation variables in the same way as the relations $(2.5 \mathrm{a})-(2.5 \mathrm{~d})$ for the unperturbed variables

$$
\delta \tilde{\boldsymbol{v}} \equiv \frac{\delta v}{v_{2}},
$$




$$
\begin{aligned}
\delta \tilde{\rho} & \equiv \frac{\delta \rho}{\rho_{2}}, \\
\delta \tilde{p} & \equiv \frac{\delta p}{p_{2}},
\end{aligned}
$$

then, the linearized perturbation equations become

$$
\begin{array}{r}
\frac{3(\gamma+1)}{4} t \frac{\partial \delta \tilde{\rho}}{\partial t}+\frac{\partial \tilde{v}}{\partial \tilde{x}} \delta \tilde{\rho}+\tilde{v} \frac{\partial \delta \tilde{\rho}}{\partial \tilde{x}}+\frac{\partial \tilde{\rho}}{\partial \tilde{x}} \delta \tilde{v}_{x}+\tilde{\rho} \tilde{\boldsymbol{v}} \cdot \delta \tilde{\boldsymbol{v}}=0, \\
\frac{3(\gamma+1)}{4} t \frac{\partial \delta \tilde{v}}{\partial t}-\frac{\gamma+1}{4} \delta \tilde{\boldsymbol{v}}+\tilde{v} \frac{\partial \delta \tilde{v}}{\partial \tilde{x}}+\frac{\partial \tilde{v}}{\partial \tilde{x}} \delta \tilde{v}_{x} \hat{\boldsymbol{x}}+\frac{\gamma-1}{2} \frac{1}{\tilde{\rho}} \tilde{\boldsymbol{\nabla}} \delta \tilde{p}-\frac{\gamma-1}{2} \frac{1}{\tilde{\rho}^{2}} \frac{\partial \tilde{p}}{\partial \tilde{x}} \delta \tilde{\rho} \hat{\boldsymbol{x}}=0,
\end{array}
$$$$
\frac{3(\gamma+1)}{4} t \frac{1}{\tilde{p}} \frac{\partial \delta \tilde{p}}{\partial t}-\frac{3(\gamma+1)}{4} t \frac{1}{\tilde{p}^{2}} \frac{\partial \tilde{p}}{\partial t} \delta \tilde{p}-\frac{3 \gamma(\gamma+1)}{4} t \frac{1}{\tilde{\rho}} \frac{\partial \delta \tilde{\rho}}{\partial t}+\frac{3 \gamma(\gamma+1)}{4} t \frac{1}{\tilde{\rho}^{2}} \frac{\partial \tilde{\rho}}{\partial t} \delta \tilde{\rho}
$$$$
+\frac{\tilde{v}}{\tilde{p}} \frac{\partial \delta \tilde{p}}{\partial \tilde{x}}-\frac{\tilde{v}}{\tilde{p}^{2}} \frac{\partial \tilde{p}}{\partial \tilde{x}} \delta \tilde{p}-\gamma \frac{\tilde{v}}{\tilde{\rho}} \frac{\partial \delta \tilde{\rho}}{\partial \tilde{x}}+\gamma \frac{\tilde{v}}{\tilde{\rho}^{2}} \frac{\partial \tilde{\rho}}{\partial \tilde{x}} \delta \tilde{\rho}+\frac{1}{\tilde{p}} \frac{\partial \tilde{p}}{\partial \tilde{x}} \delta \tilde{v}_{x}-\frac{\gamma}{\tilde{\rho}} \frac{\partial \tilde{\rho}}{\partial \tilde{x}} \delta \tilde{v}_{x}=0,
$$

where $\tilde{\nabla}$ is the normalized spatial derivative. Here we used the relations

$$
\begin{aligned}
& v_{2}=\frac{4}{3(\gamma+1)} \frac{x_{2}}{t} \propto t^{-1 / 3}, \\
& \rho_{2}=\frac{\gamma+1}{\gamma-1} \rho_{1}, \\
& p_{2}=\frac{8 \rho_{1}}{9(\gamma+1)} \frac{x_{2}^{2}}{t^{2}}
\end{aligned}
$$

from equations (2.3) and (2.4).

Since the unperturbed shock is a similarity flow, the normalized unperturbed variables $\tilde{v}, \tilde{\rho}$, and $\tilde{p}$ are a function of a single normalized similarity parameter $\tilde{x}$ :

$$
\begin{aligned}
& \tilde{v}=\tilde{v}(\tilde{x}), \\
& \tilde{\rho}=\tilde{\rho}(\tilde{x}), \\
& \tilde{p}=\tilde{p}(\tilde{x}) .
\end{aligned}
$$

Hence, we can assume that the normalized perturbation variables are composed of the similarity term, which is a function of only a similarity parameter $\tilde{x}$, the spatial dependence in Fourier series, and the time dependence in the power law $t^{s}$ :

$$
\begin{aligned}
\delta \tilde{\boldsymbol{v}} & =\delta \tilde{v}_{x}(\tilde{x}) e^{i k y} t^{s} \hat{\boldsymbol{x}}+\delta \tilde{v}_{y}(\tilde{x}) e^{i k y} t^{s} \hat{\boldsymbol{y}}, \\
\delta \tilde{\rho} & =\delta \tilde{\rho}(\tilde{x}) e^{i k y} t^{s}, \\
\delta \tilde{p} & =\delta \tilde{p}(\tilde{x}) e^{i k y} t^{s} .
\end{aligned}
$$

Since $x_{2}$ is proportional to $t^{2 / 3}$, the time derivatives of the normalized unperturbed variables are

$$
\begin{aligned}
& \frac{\partial \tilde{v}}{\partial t}=-\frac{2}{3} \frac{\tilde{x}}{t} \frac{\partial \tilde{v}}{\partial \tilde{x}}, \\
& \frac{\partial \tilde{\rho}}{\partial t}=-\frac{2}{3} \frac{\tilde{x}}{t} \frac{\partial \tilde{\rho}}{\partial \tilde{x}}, \\
& \frac{\partial \tilde{p}}{\partial t}=-\frac{2}{3} \frac{\tilde{x}}{t} \frac{\partial \tilde{p}}{\partial \tilde{x}},
\end{aligned}
$$

and the time derivatives of the normalized perturbation variables are

$$
\begin{aligned}
& \frac{\partial \delta \tilde{v}}{\partial t}=-\frac{2}{3} \frac{\tilde{x}}{t} \frac{\partial \delta \tilde{v}}{\partial \tilde{x}}+\frac{s}{t} \delta \tilde{\boldsymbol{v}}, \\
& \frac{\partial \delta \tilde{\rho}}{\partial t}=-\frac{2}{3} \frac{\tilde{x}}{t} \frac{\partial \delta \tilde{\rho}}{\partial \tilde{x}}+\frac{s}{t} \delta \tilde{\rho}, \\
& \frac{\partial \delta \tilde{p}}{\partial t}=-\frac{2}{3} \frac{\tilde{x}}{t} \frac{\partial \delta \tilde{p}}{\partial \tilde{x}}+\frac{s}{t} \delta \tilde{p}
\end{aligned}
$$


Using the above relations (4.8) and (4.9), we obtain the similarity form of the perturbation equations

$$
\begin{gathered}
\tilde{\rho} \frac{d \delta \tilde{v}_{x}}{d \tilde{x}}+\left(-\frac{\gamma+1}{2} \tilde{x}+\tilde{v}\right) \frac{d \delta \tilde{\rho}}{d \tilde{x}}+\frac{d \tilde{\rho}}{d \tilde{x}} \delta \tilde{v}_{x}+\left[\frac{3(\gamma+1)}{4} s+\frac{d \tilde{v}}{d \tilde{x}}\right] \delta \tilde{\rho}+i k x \frac{\tilde{\rho}}{\tilde{x}} \delta \tilde{v}_{y}=0, \\
\left(-\frac{\gamma+1}{2} \tilde{x}+\tilde{v}\right) \tilde{\rho} \frac{d \delta \tilde{v}_{x}}{d \tilde{x}}+\frac{\gamma-1}{2} \frac{d \delta \tilde{p}}{d \tilde{x}}+\left[\frac{\gamma+1}{4}(3 s-1)+\frac{d \tilde{v}}{d \tilde{x}}\right] \tilde{\rho} \delta \tilde{v}_{x}-\frac{\gamma-1}{2} \frac{1}{\tilde{\rho}} \frac{d \tilde{p}}{d \tilde{x}} \delta \tilde{\rho}=0, \\
\left(-\frac{\gamma+1}{2} \tilde{x}+\tilde{v}\right) \tilde{\rho} \frac{d \delta \tilde{v}_{y}}{d \tilde{x}}+i k x \frac{\gamma-1}{2} \frac{1}{\tilde{x}} \delta \tilde{p}+\frac{\gamma+1}{4}(3 s-1) \tilde{\rho} \delta \tilde{v}_{y}=0, \\
-\gamma\left(-\frac{\gamma+1}{2} \tilde{x}+\tilde{v}\right) \frac{d \delta \tilde{\rho}}{d \tilde{x}}+\frac{\tilde{\rho}}{\tilde{p}}\left(-\frac{\gamma+1}{2} \tilde{x}+\tilde{v}\right) \frac{d \delta \tilde{p}}{d \tilde{x}}+\left(-\gamma \frac{1}{\tilde{\rho}} \frac{d \tilde{\rho}}{d \tilde{x}}+\frac{1}{\tilde{p}} \frac{d \tilde{p}}{d \tilde{x}}\right) \tilde{\rho} \delta \tilde{v}_{x} \\
-\gamma\left[\frac{3(\gamma+1)}{4} s-\left(-\frac{\gamma+1}{2} \tilde{x}+\tilde{v}\right) \frac{1}{\tilde{\rho}} \frac{d \tilde{\rho}}{d \tilde{x}}\right] \delta \tilde{\rho}+\frac{\tilde{\rho}}{\tilde{p}}\left[\frac{3(\gamma+1)}{4} s-\left(-\frac{\gamma+1}{2} \tilde{x}+\tilde{v}\right) \frac{1}{\tilde{p}} \frac{d \tilde{p}}{d \tilde{x}}\right] \delta \tilde{p}=0 .
\end{gathered}
$$

Here, although we do not distinguish notationally between, say, $\delta \tilde{v}_{x}(\tilde{x})$ and $\delta \tilde{v}_{x}(\tilde{x}, y, t)$, the above equations apply to the former form.

\section{b) Spherical Shock}

If we consider the perturbation variables defined by

$$
\begin{aligned}
\delta v(r, \theta, \phi, t) & \equiv v(r, \theta, \phi, t)-v_{0}(r, t) \hat{\boldsymbol{r}}, \\
\delta \rho(r, \theta, \phi, t) & \equiv \rho(r, \theta, \phi, t)-\rho_{0}(r, t), \\
\delta p(r, \theta, \phi, t) & \equiv p(r, \theta, \phi, t)-p_{0}(r, t),
\end{aligned}
$$

and linearize the hydrodynamic equations for an adiabatic shock about the perturbation variables, we obtain the linearized perturbation equations

$$
\begin{aligned}
\frac{\partial \delta \rho}{\partial t}+\frac{1}{r^{2}} \frac{\partial\left(r^{2} v_{0} \delta \rho\right)}{\partial r}+\nabla \cdot\left(\rho_{0} \delta v\right) & =0 \\
\frac{\partial \delta v}{\partial t}+v_{0} \frac{\partial \delta v}{\partial r}+\frac{\partial v_{0}}{\partial r} \delta v_{r} \hat{r}+\frac{v_{0}}{r} \delta v_{T} & =-\frac{1}{\rho_{0}} \nabla \delta p+\frac{\delta \rho}{\rho_{0}^{2}} \frac{\partial p_{0}}{\partial r} \hat{\boldsymbol{r}}, \\
\left(\frac{\partial}{\partial t}+v_{0} \frac{\partial}{\partial r}\right)\left(\frac{\delta p}{p_{0}}-\gamma \frac{\delta \rho}{\rho_{0}}\right)+\delta v_{r} \frac{\partial}{\partial r} \log \left(\frac{p_{0}}{\rho_{0}^{\gamma}}\right) & =0 .
\end{aligned}
$$

Let us again define the normalized perturbation variables by the relations (4.3). Since the unperturbed shock is a similarity flow, the normalized unperturbed variables are

$$
\begin{aligned}
& \tilde{v}=\tilde{v}(\tilde{r}), \\
& \tilde{\rho}=\tilde{\rho}(\tilde{r}), \\
& \tilde{p}=\tilde{p}(\tilde{r}),
\end{aligned}
$$

and the normalized perturbation variables can be assumed to be

$$
\begin{aligned}
& \delta \tilde{v}=\delta \tilde{v}_{r}(\tilde{r}) Y_{l m}(\theta, \phi) t^{s} \hat{r}+\delta \tilde{v}_{T}(\tilde{r}) \tilde{r} \tilde{\nabla}_{T} Y_{l m}(\theta, \phi) t^{s} \\
& \delta \tilde{\rho}=\delta \tilde{\rho}(\tilde{r}) Y_{l m}(\theta, \phi) t^{s} \\
& \delta \tilde{p}=\delta \tilde{p}(\tilde{r}) Y_{l m}(\theta, \phi) t^{s}
\end{aligned}
$$

Here the operator $\tilde{\nabla}_{T}$ is defined by

$$
\tilde{\boldsymbol{\nabla}}_{T} \equiv \frac{1}{\tilde{r}} \frac{\partial}{\partial \theta} \hat{\boldsymbol{\theta}}+\frac{1}{\tilde{r} \sin \theta} \frac{\partial}{\partial \phi} \hat{\boldsymbol{\phi}} .
$$

Since $r_{2}$ is proportional to $t^{2 / 5}$, the time derivatives of the normalized unperturbed variables are

$$
\begin{aligned}
& \frac{\partial \tilde{v}}{\partial t}=-\frac{2}{5} \frac{\tilde{r}}{t} \frac{\partial \tilde{v}}{\partial \tilde{r}}, \\
& \frac{\partial \tilde{\rho}}{\partial t}=-\frac{2}{5} \frac{\tilde{r}}{t} \frac{\partial \tilde{\rho}}{\partial \tilde{r}}, \\
& \frac{\partial \tilde{p}}{\partial t}=-\frac{2}{5} \frac{\tilde{r}}{t} \frac{\partial \tilde{p}}{\partial \tilde{r}},
\end{aligned}
$$


and the time derivatives of the normalized perturbation variables are

$$
\begin{aligned}
& \frac{\partial \delta \tilde{v}}{\partial t}=-\frac{2}{5} \frac{\tilde{r}}{t} \frac{\partial \delta \tilde{v}}{\partial \tilde{r}}+\frac{s}{t} \delta \tilde{v}, \\
& \frac{\partial \delta \tilde{\rho}}{\partial t}=-\frac{2}{5} \frac{\tilde{r}}{t} \frac{\partial \delta \tilde{\rho}}{\partial \tilde{r}}+\frac{s}{t} \delta \tilde{\rho}, \\
& \frac{\partial \delta \tilde{p}}{\partial t}=-\frac{2}{5} \frac{\tilde{r}}{t} \frac{\partial \delta \tilde{p}}{\partial \tilde{r}}+\frac{s}{t} \delta \tilde{p}
\end{aligned}
$$

Hence, the similarity forms of the linearized perturbation equations are

$$
\begin{array}{r}
\tilde{\rho} \frac{d \delta \tilde{v}_{r}}{d \tilde{r}}+\left(-\frac{\gamma+1}{2} \tilde{r}+\tilde{v}\right) \frac{d \delta \tilde{\rho}}{d \tilde{r}}+\left(2 \frac{\tilde{\rho}}{\tilde{r}}+\frac{d \tilde{\rho}}{d \tilde{r}}\right) \delta \tilde{v}_{r}+\left[\frac{5(\gamma+1)}{4} s+2 \frac{\tilde{v}}{\tilde{r}}+\frac{d \tilde{v}}{d \tilde{r}}\right] \delta \tilde{\rho}-l(l+1) \frac{\tilde{\rho}}{\tilde{r}} \delta \tilde{v}_{T}=0, \\
\left(-\frac{\gamma+1}{2} \tilde{r}+\tilde{v}\right) \tilde{\rho} \frac{d \delta \tilde{v}_{r}}{d \tilde{r}}+\frac{\gamma-1}{2} \frac{d \delta \tilde{p}}{d \tilde{r}}+\left[\frac{\gamma+1}{4}(5 s-3)+\frac{d \tilde{v}}{d \tilde{r}}\right] \tilde{\rho} \delta \tilde{v} r-\frac{\gamma-1}{2} \frac{1}{\tilde{\rho}} \frac{d \tilde{p}}{d \tilde{r}} \delta \tilde{\rho}=0, \\
\left(-\frac{\gamma+1}{2} \tilde{r}+\tilde{v}\right) \tilde{\rho} \frac{d \delta \tilde{v}}{d \tilde{r}}+\frac{\gamma-1}{2} \frac{1}{\tilde{r}} \delta \tilde{p}+\left[\frac{\gamma+1}{4}(5 s-3)+\frac{\tilde{v}}{\tilde{r}}\right] \tilde{\rho} \delta \tilde{v}_{T}=0, \\
-\gamma\left(-\frac{\gamma+1}{2} \tilde{r}+\tilde{v}\right) \frac{d \delta \tilde{\rho}}{d \tilde{r}}+\frac{\tilde{\rho}}{\tilde{p}}\left(-\frac{\gamma+1}{2} \tilde{r}+\tilde{v}\right) \frac{d \delta \tilde{p}}{d \tilde{r}}+\left(-\gamma \frac{1}{\tilde{\rho}} \frac{d \tilde{\rho}}{d \tilde{r}}+\frac{1}{\tilde{p}} \frac{d \tilde{p}}{d \tilde{r}}\right) \tilde{\rho} \delta \tilde{v}_{r} \\
-\gamma\left[\frac{5(\gamma+1)}{4} s-\left(-\frac{\gamma+1}{2} \tilde{r}+\tilde{v}\right) \frac{1}{\tilde{\rho}} \frac{d \tilde{\rho}}{d \tilde{r}}\right] \delta \tilde{\rho}+\frac{\tilde{\rho}}{\tilde{p}}\left[\frac{5(\gamma+1)}{4} s-\left(-\frac{\gamma+1}{2} \tilde{r}+\tilde{v}\right) \frac{1}{\tilde{p}} \frac{d \tilde{p}}{d \tilde{r}}\right] \delta \tilde{p}=0,
\end{array}
$$

where the relations

$$
\begin{aligned}
& v_{2}=\frac{4}{5(\gamma+1)} \frac{r_{2}}{t} \propto t^{-3 / 5}, \\
& \rho_{2}=\frac{\gamma+1}{\gamma-1} \rho_{1},
\end{aligned}
$$

and

$$
p_{2}=\frac{8 \rho_{1}}{25(\gamma+1)} \frac{r_{2}^{2}}{t^{2}},
$$

from equations (2.3) and (2.8), are used. Obviously, the above equations apply to, for example, $\delta \tilde{v}_{r}(\tilde{r})$, and not $\delta \tilde{v}_{r}(\tilde{r}, \theta, \phi, t)$. The third and fourth terms of the first equation and the third term of the third equation contain the curvature effect of the spherical geometry.

\section{BOUNDARY CONDITIONS}

\section{a) Plane-Parallel Shock}

Let us consider the boundary conditions at the shock front when the shock is perturbed (Welter 1982). For the gas flow through the shock front, we require the conservations of mass, momentum, and energy. Hence, the boundary conditions for a perturbed plane-parallel adiabatic shock are

$$
\begin{aligned}
{\left[\rho u_{x}\right] } & =0, \\
{\left[p+\rho u_{x}^{2}\right] } & =0, \\
{\left[u_{y}\right] } & =0, \\
{\left[\frac{1}{2} u^{2}+\frac{\gamma}{\gamma-1} \frac{p}{\rho}\right] } & =0 .
\end{aligned}
$$

Here, $u_{x}$ is the gas velocity relative to and perpendicular to the shock front, $u_{y}$ is the gas velocity parallel to the shock front, and the square brackets denote the difference in the enclosed quantity across the shock front. 
By linearizing the boundary conditions with respect to the perturbed quantities, equations (5.1a), (5.1b), and (5.1d) become

$$
\begin{array}{r}
\left(\delta \rho+\eta \frac{\partial \rho_{0}}{\partial x}\right)\left(v_{0}-u_{1}\right)+\rho_{0}\left(\delta v_{x}+\eta \frac{\partial v_{0}}{\partial x}-\frac{d \eta}{d t}\right)=-\rho_{1} \frac{d \eta}{d t} \\
\left(\delta p+\eta \frac{\partial p_{0}}{\partial x}\right)+\left(\delta \rho+\eta \frac{\partial \rho_{0}}{\partial x}\right)\left(v_{0}-u_{1}\right)^{2}+2 \rho_{0}\left(v_{0}-u_{1}\right)\left(\delta v_{x}+\eta \frac{\partial v_{0}}{\partial x}-\frac{d \eta}{d t}\right)=2 \rho_{1} u_{1} \frac{d \eta}{d t} \\
\left(v_{0}-u_{1}\right)\left(\delta v_{x}+\eta \frac{\partial v_{0}}{\partial x}-\frac{d \eta}{d t}\right)+\frac{\gamma}{\gamma-1} \frac{1}{\rho_{0}}\left(\delta p+\eta \frac{\partial p_{0}}{\partial x}\right)-\frac{\gamma}{\gamma-1} \frac{p_{0}}{\rho_{0}^{2}}\left(\delta \rho+\eta \frac{\partial \rho_{0}}{\partial x}\right)=u_{1} \frac{d \eta}{d t}
\end{array}
$$

where $\eta$ is the perturbation of the position of the shock front. Here, the perturbation of quantities in the ambient gas is neglected.

Let us define the normalized perturbation of the position of the shock front by

$$
\tilde{\eta} \equiv \frac{\eta}{x_{2}}
$$

and assume the spatial dependence in Fourier series and the time dependence in power law

$$
\tilde{\eta}=\tilde{\eta} e^{i k y} t^{s} .
$$

Then equations $(5.2 \mathrm{a})-(5.2 \mathrm{c})$ become

$$
\begin{aligned}
\delta \tilde{v}_{x} & =-\tilde{\eta} \frac{d \tilde{v}}{d \tilde{x}}+\tilde{\eta}(1+1.5 s), \\
\delta \tilde{\rho} & =-\tilde{\eta} \frac{d \tilde{\rho}}{d \tilde{x}}, \\
\delta \tilde{p} & =-\tilde{\eta} \frac{d \tilde{p}}{d \tilde{x}}+\tilde{\eta}(2+3 s),
\end{aligned}
$$

where the relations $\tilde{v}=\tilde{\rho}=\tilde{p}=1$ and $x=x_{2}$ at the shock front and $x_{2} \propto t^{2 / 3}$ are used. In equations (5.4a)-(5.4c), we see that $\tilde{\eta}$ is a scale factor and can have an arbitrary value. If we assume for convenience $\tilde{\eta}=1$, we obtain the normalized boundary conditions of the $x$-component of velocity, density, and pressure perturbations at the shock front

$$
\begin{aligned}
\delta \tilde{v}_{x} & =-\frac{d \tilde{v}}{d \tilde{x}}+1+1.5 s \\
\delta \tilde{\rho} & =-\frac{d \tilde{\rho}}{d \tilde{x}} \\
\delta \tilde{p} & =-\frac{d \tilde{p}}{d \tilde{x}}+2+3 s
\end{aligned}
$$

From condition (5.1c), the $y$-component of the velocity of the gas flow at $k y=\pi / 2$ just behind the shock front becomes

$$
u_{y}=\frac{2}{\gamma+1} u_{1} \cos \theta \sin \theta
$$

where $\theta$ is the angle between the shock front and the plane with $x=x_{2}$ at $k y=\pi / 2$. Since the angle $\theta$ is small in the linear regime of the perturbation, we know approximately

$$
\begin{aligned}
& \cos \theta \approx 1, \\
& \sin \theta \approx k \eta .
\end{aligned}
$$

Hence, again by assuming $\tilde{\eta}=1$, we obtain the normalized boundary condition of the $y$-component velocity perturbation at the shock front

$$
\delta \tilde{v}_{y}=-i k x
$$

For the boundary condition at the origin, we take the requirement that the fluid should not undergo divergent perturbations in the Lagrangian sense at the origin (i.e., the fractional density change of the displaced volume element goes to zero). Then from the perturbation equation of the continuity equation (2.1a),

$$
\frac{D}{D t}\left(\frac{\delta \rho}{\rho}\right)+\nabla \cdot \delta v=0,
$$


where $D / D t$ is the total derivative with respect to time in the Lagrangian coordinates, we obtain a condition at the origin

$$
\nabla \cdot \delta \boldsymbol{v}=0 \text {. }
$$

From the perturbation equation of energy equation (2.1c)

condition (5.10) gives

$$
\frac{\partial \delta p}{\partial t}+\delta v \cdot \nabla p+v \cdot \nabla \delta p+\gamma \delta p \nabla \cdot v+\gamma p \nabla \cdot \delta v=0
$$

$$
\frac{\partial \delta p}{\partial t}+\delta v \cdot \nabla p+v \cdot \nabla \delta p+\gamma \delta p \nabla \cdot v=0
$$

at the origin. Since at the origin

$$
\delta v \cdot \nabla p \propto v \cdot \nabla \delta p \propto \delta p \nabla \cdot v \propto \delta p,
$$

the boundary condition for an adiabatic shock at the origin is

$$
\delta p=0 .
$$

One might think that the correct boundary condition at the origin is $\delta v_{x} \propto x^{a}$ with $a \geq 1$, since $v_{0} \approx x$ near the origin. However, for any $s$ the perturbed velocity will not satisfy this condition near the origin. This is because the temperature singularity $(T \rightarrow \infty)$ at the origin tends to lead to divergent velocity perturbations, even when the perturbation is physically reasonable. In order for the fluid motion to be physically reasonable at the origin, it is necessary that the energy perturbation should vanish at the origin. Since the energy perturbation goes as $\delta p, v^{2} \delta \rho$, or $v \rho \delta v$, again, we can obtain the same boundary condition $\delta p=0$ at the origin.

\section{b) Spherical Shock}

From conservations of mass, momentum, and energy, the boundary conditions at the shock front for a perturbed spherical adiabatic shock can be written as

$$
\begin{aligned}
{\left[\rho u_{r}\right] } & =0, \\
{\left[p+\rho u_{r}^{2}\right] } & =0, \\
{\left[u_{T}\right] } & =0, \\
{\left[\frac{1}{2} u^{2}+\frac{\gamma}{\gamma-1} \frac{p}{\rho}\right] } & =0 .
\end{aligned}
$$

Here, $u_{r}$ is the radial gas velocity relative to the shock front and $u_{T}$ is the gas velocity tangential to the shock front.

If we assume that the normalized perturbation in the position of the shock front $\tilde{\eta}$ has spatial dependence proportional to a spherical harmonic and time dependence proportional to a power of $t$

$$
\begin{aligned}
\tilde{\eta} & \equiv \frac{\eta}{r_{2}} \\
& =\tilde{\eta} Y_{l m}(\theta, \phi) t^{s},
\end{aligned}
$$

and the scale factor $\tilde{\eta}$ equals 1 , then we obtain the normalized boundary conditions of the perturbation quantities at the shock front

$$
\begin{aligned}
\delta \tilde{v}_{r} & =-\frac{d \tilde{v}}{d \tilde{r}}+1+2.5 s, \\
\delta \tilde{\rho} & =-\frac{d \tilde{\rho}}{d \tilde{r}}, \\
\delta \tilde{p} & =-\frac{d \tilde{p}}{d \tilde{r}}+2+5 s, \\
\delta \tilde{v}_{T} & =-1 .
\end{aligned}
$$

By the same argument as for the plane-parallel case, the boundary condition at the origin is

$$
\delta p=0 \text {. }
$$

\section{NUMERICAL SOLUTIONS}

From equations (4.10a)-(4.10d) for the plane-parallel shock or from equations (4.18a)-(4.18d) for the spherical shock, the derivatives of the normalized perturbation variables with respect to the normalized similarity parameter are calculated

$$
\frac{d \delta \tilde{\boldsymbol{Q}}}{d \tilde{x}}=\mathbf{A} \cdot \delta \tilde{\boldsymbol{Q}}
$$

\section{(C) American Astronomical Society - Provided by the NASA Astrophysics Data System}




$$
\frac{d \delta \tilde{\boldsymbol{Q}}}{d \tilde{r}}=\mathbf{A} \cdot \delta \tilde{\boldsymbol{Q}}
$$

Here, $\delta \tilde{\boldsymbol{Q}}$ is a vector with

$$
\delta \tilde{\boldsymbol{Q}}=\left(\delta \tilde{v}_{x}, \delta \tilde{\rho}, \delta \tilde{p}, \delta \tilde{v}_{y}\right)
$$

or

$$
\delta \tilde{\boldsymbol{Q}}=\left(\delta \tilde{v}_{r}, \delta \tilde{\rho}, \delta \tilde{p}, \delta \tilde{v}_{T}\right),
$$

and $\mathbf{A}$ is a $4 \times 4$ matrix whose elements are a function of $\tilde{v}, \tilde{\rho}, \tilde{p}$, and $\tilde{x}$ (or $\tilde{r})$ for given values of $\gamma, k x$ (or $l$ ), and $s$. By a finite difference formula

$$
\frac{d \delta \tilde{Q}(\tilde{x})}{d \tilde{x}}=\frac{\delta \tilde{Q}(\tilde{x}+h)-\delta \tilde{Q}(\tilde{x})}{h}
$$

where $h$ is a finite step size, if we know the values of the perturbation variables at a given value of $\tilde{x}$, then we can compute the values of the perturbation variables at the next value of $\tilde{x}+h$.

For given values of $\gamma$ and $k x$ (or $l$ ), we first guess the eigenvalue $s$. Using the boundary conditions at the shock front (5.5) and (5.8) or (5.17), we integrate numerically the perturbation equations from $\tilde{x}=1$ to $\tilde{x}=0$ with $h=-0.001$, and we check whether the result satisfies the boundary condition at the origin (5.14) or (5.18). If the result does not satisfy the boundary condition at the origin, we modify $s$ to a better value $s+\Delta s$ by the formula

$$
\Delta s=-\frac{\delta \tilde{p}(\tilde{x}=0)}{\partial \delta \tilde{p}(\tilde{x}=0) / \partial s}
$$

where $\partial \delta \tilde{p} / \partial s$ is calculated from

$$
\frac{d}{d \tilde{x}} \frac{\partial \delta \tilde{\boldsymbol{Q}}}{\partial s}=\frac{\partial \mathbf{A}}{\partial s} \cdot \delta \tilde{\boldsymbol{Q}}+\mathbf{A} \cdot \frac{\partial \delta \tilde{\boldsymbol{Q}}}{\partial s},
$$

with the finite difference method. When $|\Delta s| \leq 10^{-4}$, we finish the iteration and take $s$ as the solution for given values of $\gamma$ and $k x$ (or l). The results of numerical calculations, the frequency spectra of the real and imaginary parts of $s$ for several values of $\gamma$ which correspond to the increasing mode of $s$ for $\gamma=1$ in $\S$ III, are shown in Figures 5 and 6 for the plane-parallel shock and in Figures 7 and 8 for the spherical shock.

If we assume that $\delta p \propto x^{a}$ (or $\delta p \propto r^{a}$ ) around the origin, we obtain four $a$ 's from the secular equation of the perturbation equations (4.10) or (4.18). In the plane-parallel shock, for $k x \rightarrow 0$ we have

$$
\begin{aligned}
& a_{1}=\frac{\gamma}{\gamma-1}, \\
& a_{2}= \begin{cases}-\epsilon & \left(s \lesssim-\frac{2}{3} \frac{2 \gamma-1}{\gamma} \text { or } s \gtrsim-\frac{1}{3}\right), \\
+\epsilon & \left(-\frac{2}{3} \frac{2 \gamma-1}{\gamma} \lesssim s \lesssim-\frac{1}{3}\right),\end{cases} \\
& a_{3}=\frac{\gamma}{\gamma-1}\left(\frac{3}{2} s+1\right)+1 \text {, } \\
& a_{4}=\frac{1}{2} \frac{\gamma}{\gamma-1}(3 s+1),
\end{aligned}
$$

where $\epsilon$ is a small real number, and for $k x \rightarrow \infty$ we have roughly

$$
\begin{aligned}
& a_{1} \approx k x, \\
& a_{2} \approx-k x, \\
& a_{3} \approx \frac{\gamma}{\gamma-1}\left(\frac{3}{2} s+\frac{5}{4}\right)+i C, \\
& a_{4} \approx \frac{\gamma}{\gamma-1}\left(\frac{3}{2} s+\frac{5}{4}\right)-i C,
\end{aligned}
$$



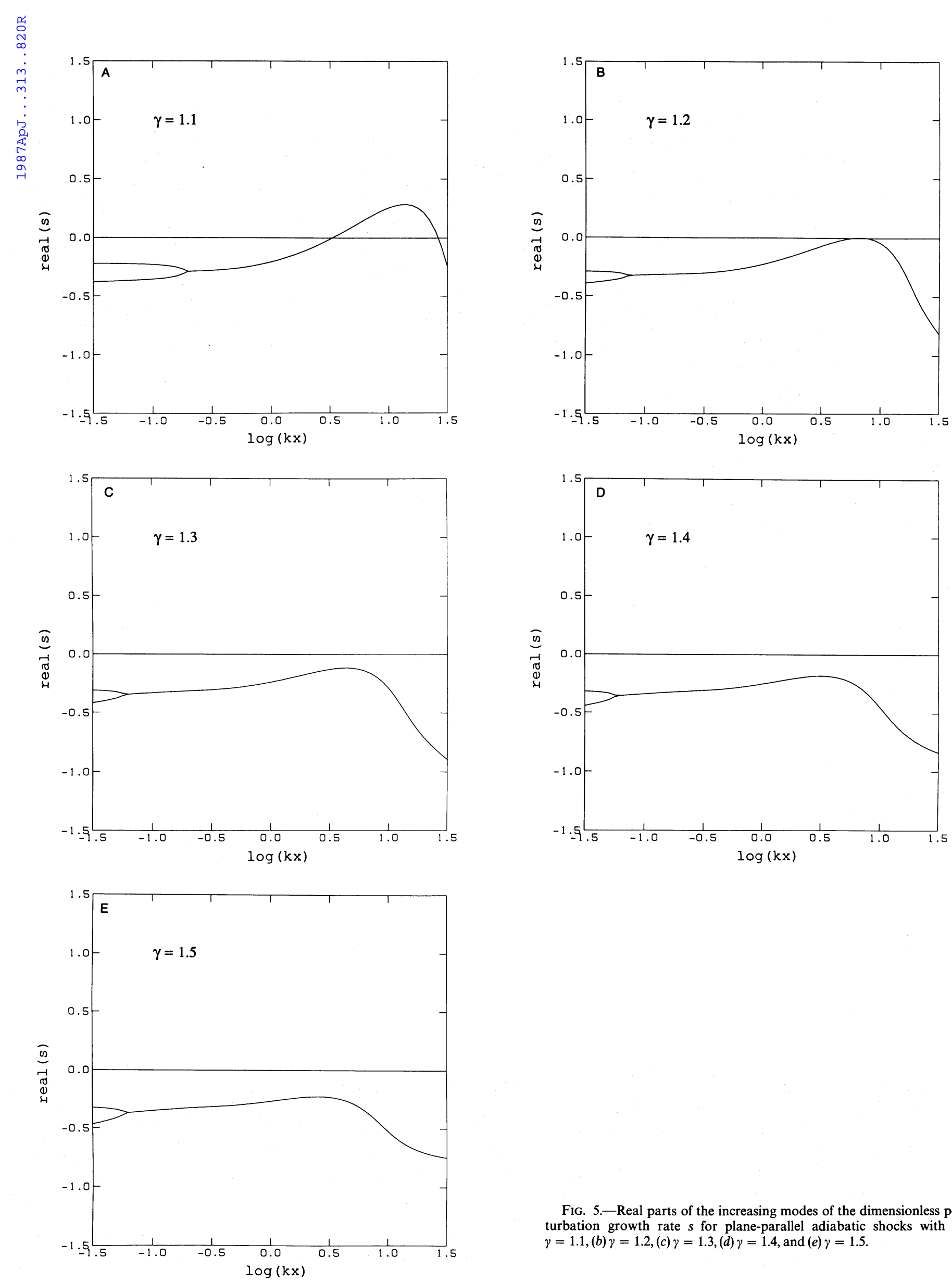

Fig. 5.- Real parts of the increasing modes of the dimensionless perturbation growth rate $s$ for plane-parallel adiabatic shocks with $(a)$ $\gamma=1.1,(b) \gamma=1.2,(c) \gamma=1.3,(d) \gamma=1.4$, and $(e) \gamma=1.5$. 

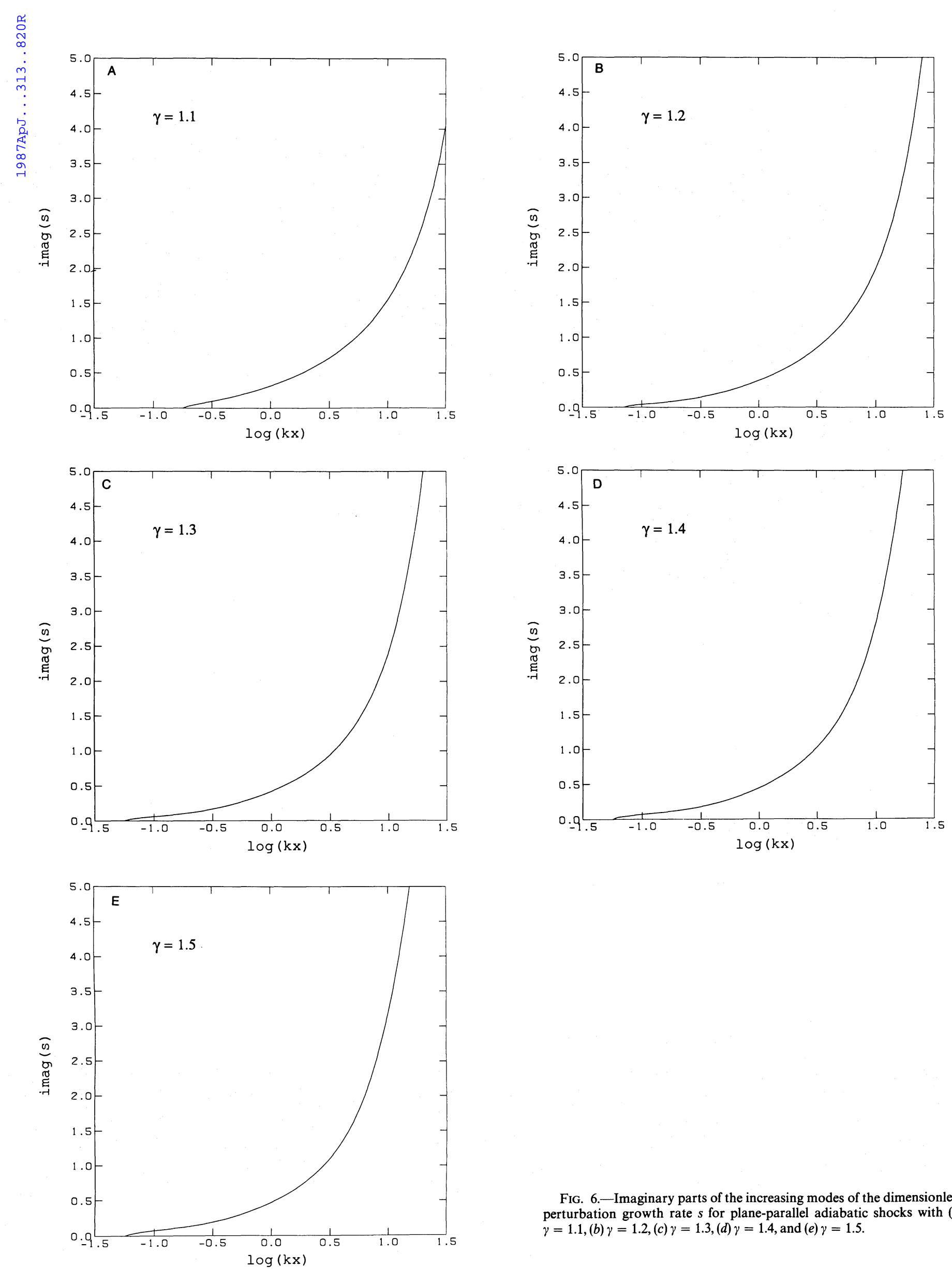

FIG. 6.-Imaginary parts of the increasing modes of the dimensionless perturbation growth rate $s$ for plane-parallel adiabatic shocks with $(a)$ $\gamma=1.1,(b) \gamma=1.2,(c) \gamma=1.3,(d) \gamma=1.4$, and $(e) \gamma=1.5$. 

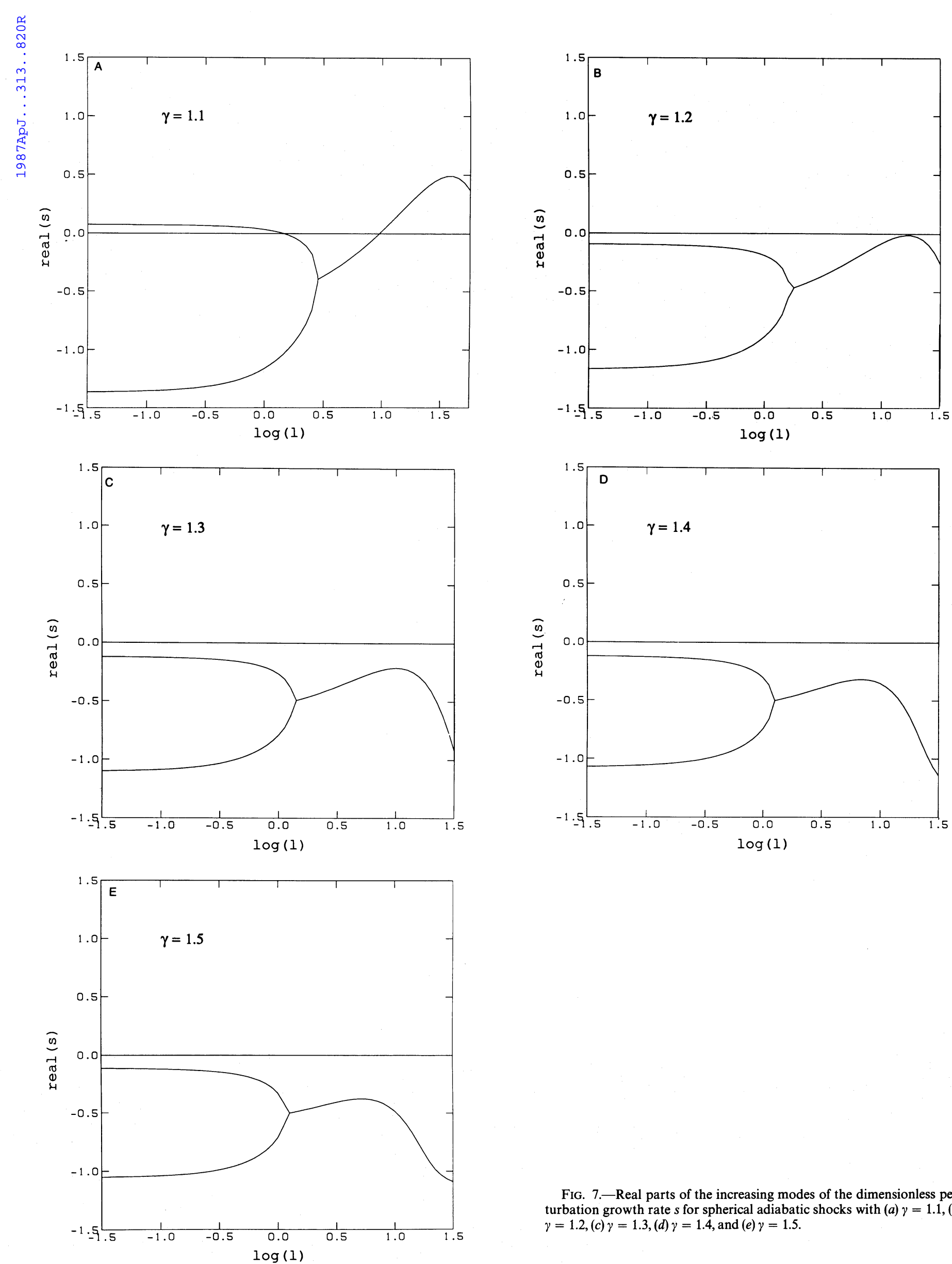

FIG. 7.-Real parts of the increasing modes of the dimensionless perturbation growth rate $s$ for spherical adiabatic shocks with $(a) \gamma=1.1,(b)$ $\gamma=1.2,(c) \gamma=1.3,(d) \gamma=1.4$, and $(e) \gamma=1.5$. 

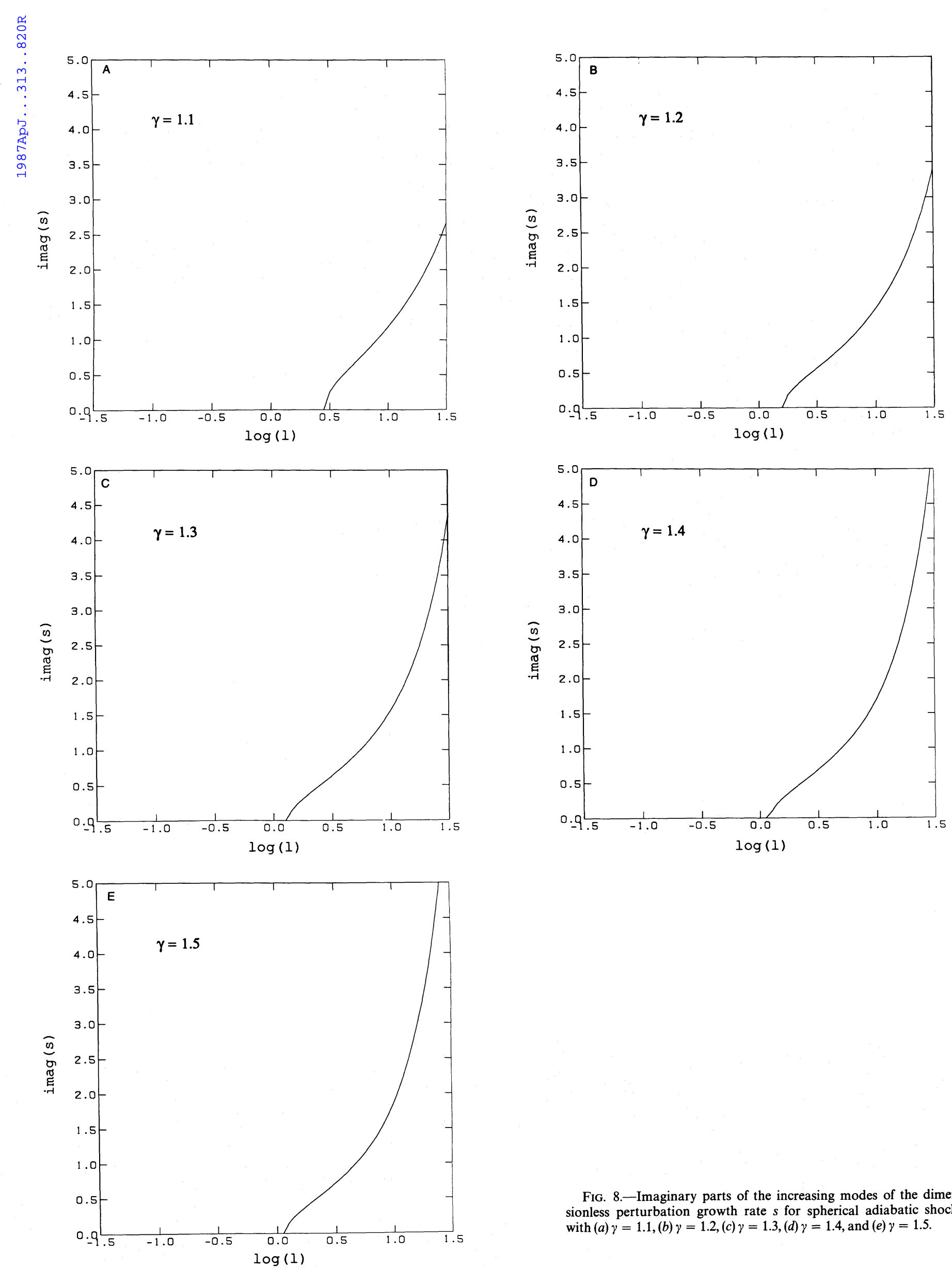

FIG. 8.-Imaginary parts of the increasing modes of the dimensionless perturbation growth rate $s$ for spherical adiabatic shocks with $(a) \gamma=1.1,(b) \gamma=1.2,(c) \gamma=1.3,(d) \gamma=1.4$, and $(e) \gamma=1.5$. 
where $C$ is a real number. In the spherical shock, for $l \rightarrow 0$ we have

$$
\begin{aligned}
& a_{1}=\frac{4-\gamma}{\gamma-1}, \\
& a_{2}= \begin{cases}-\epsilon & \left(s \lesssim-\frac{2}{5} \frac{2 \gamma+1}{\gamma} \text { or } s \gtrsim \frac{\gamma-6}{5 \gamma}\right), \\
+\epsilon & \left(-\frac{2}{5} \frac{2 \gamma+1}{\gamma} \lesssim s \lesssim \frac{\gamma-6}{5 \gamma}\right),\end{cases} \\
& a_{3}=\frac{1}{\gamma-1}\left(\frac{5}{2} \gamma s+\gamma+2\right)+1, \\
& a_{4}=\frac{1}{\gamma-1}\left(\frac{5}{2} \gamma s-\frac{1}{2} \gamma+3\right),
\end{aligned}
$$

and for $l \rightarrow \infty$ we have again roughly

$$
\begin{aligned}
& a_{1} \approx l, \\
& a_{2} \approx-l, \\
& a_{3} \approx \frac{1}{\gamma-1}\left(\frac{5}{2} \gamma s+\frac{3}{4} \gamma+2\right)+i C, \\
& a_{4} \approx \frac{1}{\gamma-1}\left(\frac{5}{2} \gamma s+\frac{3}{4} \gamma+2\right)-i C .
\end{aligned}
$$

In order to guarantee the existence of the eigenvalue $s$ which satisfies the inner boundary condition $\delta p=0$, we expect that there should be only one $a$ with a negative real part. The eigenvalues $s$ which correspond to the increasing mode of $s$ for $\gamma=1$ will have only one $a$ with a negative real part, if

$$
\operatorname{Real}(s) \gtrsim-\frac{2}{3} \frac{2 \gamma-1}{\gamma}, \quad \text { for } k x \rightarrow 0
$$

and

$$
\text { Real }(s) \gtrsim-\frac{5}{6}, \quad \text { for } k x \rightarrow \infty,
$$

in the plane-parallel shock, and if

$$
\operatorname{Real}(s) \gtrsim-\frac{2}{5} \frac{2 \gamma+1}{\gamma}, \quad \text { for } l \rightarrow 0,
$$

and

$$
\operatorname{Real}(s) \gtrsim-\frac{3}{10}-\frac{4}{5} \frac{1}{\gamma}, \quad \text { for } l \rightarrow \infty,
$$

in the spherical shock. Hence, we can find the increasing mode. But the eigenvalues $s$ which correspond to the decaying mode of $s$ for $\gamma=1$ always have three $a$ 's with a negative real part, so we cannot find the decaying mode with the similarity assumption in the perturbation quantities. This argument also explains the flattening of the frequency spectra of the increasing mode for small wavelengths.

The singular behavior of the decaying mode is somewhat surprising, since this mode appears well behaved in the thin shell approximation. The singular behavior of such solutions as $t \rightarrow 0$ appears to induce singular behavior at the origin even at late times.

We see in the frequency spectra that the adiabatic shock becomes stable against dynamic perturbation for small wavelengths. The reason is that for the small wavelengths in which the thickness of the shell cannot be ignored, the restoring force by the pressure perturbation prevents the perturbation from growing.

The plane-parallel shock is overstable when $\gamma \lesssim 1.2$, which corresponds to an ideal gas with internal degree of freedom $f \gtrsim 10$. The internal degree of freedom $f$ in an ideal gas is related to an adiabatic index $\gamma$ by

$$
\gamma=1+\frac{2}{f} .
$$

The plane-parallel shock with $\gamma=1.2$ is marginally overstable around the wavelengths corresponding to $k x \approx 7$. The plane-parallel shock with $\gamma=1.1$ is overstable for the wavelengths greater than that corresponding to $k x \approx 25$ and is most overstable for a wavelength corresponding to $k x \approx 14$.

\section{(C) American Astronomical Society - Provided by the NASA Astrophysics Data System}


The spherical shock is overstable when $\gamma<1.2$, which roughly corresponds to an ideal gas with the internal degree of freedom $f>10$. The spherical shock with $\gamma=1.1$ is shown to be slightly unstable for the region of wavelengths with $l \lesssim 1$. But since this region of wavelengths does not have any physical meaning, the unexpected behavior of instability spectrum for $\gamma=1.1$, which comes from the numerical error, does not change our results. The spherical shock with $\gamma=1.1$ has the wavelength of maximum overstability corresponding to $l \approx 40$ and the minimum wavelength of overstability corresponding to $l \approx 80$. When compared to the thickness $h$ of the shell, the minimum wavelength for $\gamma=1.1$ corresponds to

$$
\begin{aligned}
k h & \approx \frac{l}{3} \frac{\gamma-1}{\gamma+1} \\
& \approx 1.27 .
\end{aligned}
$$

Therefore, we can roughly say that the adiabatic shock becomes stable against the dynamic perturbations whose wavelengths are comparable to the thickness of the shell or smaller.

In order to check the accuracy of the above calculations using the finite difference formula, we integrated numerically the perturbation equations by Adam's predictor-corrector method using the subroutine DGEAR in IMSL (International Mathematics and Statistics Library) and found eigenvalues $s$ for several values of $\gamma$ and $k x$. From this calculation, we confirmed the general behavior of the frequency spectra even though the errors become larger for smaller $\gamma$ and $k x$.

The reason that the numerical inaccuracy grows for smaller $k x$ (or $l$ ) is that the divergent term of $\delta p$ whose coefficient we want to make zero becomes only weakly divergent around the origin for small $k x$ (or $l$ ). Therefore, the fluid motion around the origin which should be determined by the perturbation at large distance from the origin can be affected by the numerical error around the origin. The reason for the larger numerical error for smaller $\gamma$ is that the unperturbed density goes to zero faster near the origin for smaller $\gamma$.

Until now, we have considered the somewhat idealized case of an adiabatic shock without cooling, conductivity, viscosity, or magnetic field. If the radiative cooling is considered, it will reduce the temperature and the thickness of the shell. Hence the effective value of $\gamma$ will decrease, even though a shock with the radiative cooling is expected to have an internal structure different from the structure of an adiabatic shock with $\gamma$ close to 1 . For example, in an adiabatic shock with $\gamma$ close to 1 , the ratio of the internal thermal pressure $p_{i}$ to the external ram pressure $p_{e}, p_{i} / p_{e}$, approaches $\frac{1}{2}$, whereas in an isothermal shock with $\gamma$ close to 1 the ratio approaches $\frac{1}{6}$. For the plane-parallel case, the radius of an adiabatic shock expands as $t^{2 / 3}$, whereas that of an isothermal shock expands as $t^{6 / 11}$. For the spherical case, the radius of an adiabatic shock expands as $t^{2 / 5}$, whereas that of an isothermal shock expands as $t^{2 / 7}$. But since the overstability of a shock mainly depends on the thickness of the shell, a shock with radiative cooling will become overstable for dynamic perturbations, and the wavelength in which the shock is the most overstable will decrease.

\section{CONCLUSIONS}

Our basic result is that the dynamic overstability for a thin shock, described previously by Vishniac (1983), exists in cases where the dense postshock layer has a finite width. What does this imply for more realistic shocks in the interstellar medium? First, we note that our linear analysis is strictly applicable only to an adiabatic shock wave. To the extent that shocked gas in the interstellar medium is adiabatic, it will have $\gamma=5 / 3$, and any shock wave will be stable. On the other hand, when radiative processes become important in the postshock layer, the gas will act as though it had an effective $\gamma$ close to one. Second, our analysis is purely linear. When a shock is unstable in the linear regime, we expect that perturbations will grow until nonlinear effects are important. Such effects are beyond the scope of this paper. However, we note that we can expect this process to lead to the fragmentation of the shock. Interactions between such fragments and the possible growth of gravitationally bound structures would seem to require a general three-dimensional treatment. In such a case the role of the interstellar magnetic field, neglected here, would have to be explored in order to achieve realistic results.

Besides the importance of these results as proof of the existence of the dynamic instability for a realistic shock, there is an immediate application for this work. Three-dimensional models of shock waves are now being used to explore the fragmentation of a cooling shock (e.g., Rozyczka 1985). We suggest that the results of these calculations are not believable unless they can show the dynamical evolution of linear perturbations that agree with our work in the appropriate limit.

What can we say about the astrophysical applications of this work? Keeping in mind that this analysis has all the limitations expected of a linear calculation, two points still stand out. First, the fact that a shock wave fragments during cooling raises the possibility of calculating the dominant scale of fragmentation. Using the small wavelength cutoff suggested by this work it should be possible to estimate that length from one-dimensional calculations of a cooling shock wave. Second, confirmation of the overstability shows one way that large-scale pressure-driven motions in the intergalactic medium can feed small-scale structure. Recent work by Bust and Vishniac (1986) uses similar arguments to support the idea that the Lyman- $\alpha$ clouds could be the result of the fragmentation of intergalactic shock waves.

We wish to thank Edmund Bertschinger for his comments. This work was supported by the Exxon Educational Foundation and the National Science Foundation grant AST 84-51736.

Bernstein, I. R., and Book, D. 1980, Ap. J., 240, 223. Bertschinger, E. 1983, Ap. J., 268, 17. Bertschinger, E. 1983, Ap. J., Bust, G., and Vishniac, E. T. 1986, Ap. J., submitted.

\section{REFERENCES}

Cheng, A. 1979, Ap. J., 227, 955.

Elmegreen, B. G., and Elmegreen, D. M. 1978, Ap. J., 220, 1051.

Elmegreen, B. G., and Lada, C. J. 1977, Ap. J., 214, 725.

Erpenbeck, J. J. 1962, Phys. Fluids, 5, 1181.

\section{(C) American Astronomical Society - Provided by the NASA Astrophysics Data System}


Gaffet, B. 1984a, Ap. J., 279, 419.

Ikeuchi, S. 1981, Pub. Astr. Soc. Japan, 33, 211.
Ikstr. Ap., 135, 94.

I Ikeuchi, S., Tomisaka, K., and Ostriker, J. P. 1983, Ap. J., 265, 583.

Isenberg, P. A. 1977, Ap. J., 217, 597.

I I Landau, L. D., and Lifshitz, E. M. 1959, Fluid Mechanics (New York:

Pergamon).

Newman, W. I. 1980, Ap. J., 236, 880.
Ostriker, J. P., and Cowie, L. L. 1981, Ap. J. (Letters), 243, L127.

Rozyczka, M. 1985, Astr. Ap., 143, 59.

Spitzer, L. 1978, Physical Processes in the Interstellar Medium (New York: Wiley-Interscience).

Vishniac, E. T. 1983, Ap. J., 274, 152.

Welter, G. L. 1982, Astr. Ap., 105, 237.

Welter, G. L., and Schmid-Burgk, J. 1981, Ap. J., 245, 927.

Dongsu Ryu and Ethan T. VishniaC: Department of Astronomy, University of Texas, Austin, TX 78712 